\title{
Conformal Geometric Algebra for Endoscope-Traking System Calibration in Neurosurgery
}

\author{
Silena Herold-García ${ }^{1}$, Jorge Rivera-Rovelo ${ }^{2}$, and Eduardo Bayro-Corrochano ${ }^{2}$ \\ ${ }^{1}$ Universidad de Oriente, Santiago de Cuba \\ silena@csd.uo.edu.cu \\ ${ }^{2}$ CINVESTAV del IPN, Unidad Guadalajara, \\ Department of Electric Engineering and Computer Sciences, \\ Av. Científica 1145, El Bajío, Zapopan, Jalisco, México \\ $\{$ rivera, edb\}@gdl. cinvestav . $m x$
}

\begin{abstract}
One necessary task in the operating room is to establish a common reference frame, in order to relate the information obtained from different sensors, and to combine both the preoperative with the intraoperative information. To estimate the transformations between different data, fiducial markers are typically used. In this paper we present a formulation of the known hand-eye calibration problem, to estimate the transformation between an endoscopic camera and the set of spherical markers placed on it, using the conformal geometric algebra framework. Such markers are tracked by an optical stereo tracking system, which help to relate the real world with the virtual model created before surgery. Experimental results shows that our method is reliable and useful for medical applications in real time like neurosurgery.
\end{abstract}

Keywords: Hand-Eye Calibration, Geometric Algebra, Neurosurgery, Endoscope Calibration.

\section{Introduction}

In general, the registration process consists in the estimation of a common geometric reference frame between two or more data sets. These data sets can be data taken using different modalities, or the same modality but in different times. In surgery, the registration is made with the purpose of having more preoperative and intraoperative information for diagnostic and navigation. That is, registration helps to relate the positions of surgical instruments tracked in real time by an optical tracking system, with the virtual model created with preoperative images. In the operating room, there are multiple local coordinates systems that must be related in order to show to the surgeon a virtual model of what is happening in the real world. The figure 1 illustrates the scenario. When using information obtained from endoscopy or neuro-sonography, we must relate what is been observed by the endoscopic camera (or ultrasound system), with 


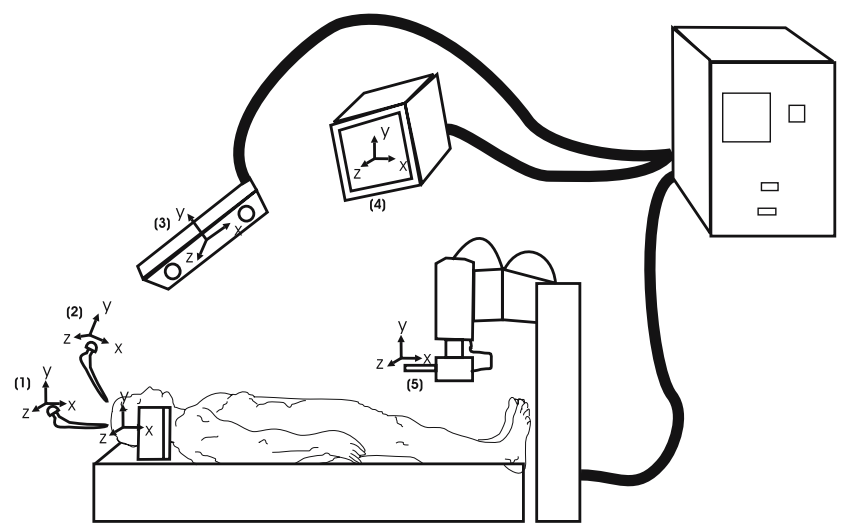

Fig. 1. Some of the different coordinates systems present in the operating room: surgical instruments (1,2), optical tracking system (3), virtual model(4), endoscopy (5)

the virtual model. Therefore, calibration techniques are needed to register all the equipment needed in surgery.

In this paper we are proposing a formulation of the hand-eye calibration problem, which is known in robotics community [3456], but in terms of the Conformal Geometric Algebra in order to calculate the transformation (rotation and translation) between the coordinate system of the endoscopic camera, and the coordinate system of the spherical markers placed to the endoscope. We call this task the endoscope-tracking calibration. The proposed method in this paper in contrast to [3], computes both rotation and translation, at the same time; in addition to, our formulation avoids to take care of certain particularities which we need to take into account in other methods [456], making simpler to understand and to compute them.

\section{Geometric Algebra}

The Geometric Algebra $G_{p, q, r}$ is constructed over the vector space $\mathcal{V}^{p, q, r}$, where $p, q, r$ denote the signature of the algebra; if $p \neq 0$ and $q=r=0$, the metric is Euclidean; if only $r=0$, the metric is pseudo euclidean; if $p \neq 0, q \neq 0, r \neq 0$, the metric is degenerate. In this algebra, we have the geometric product which is defined as in (1) for two vectors $a, b$, and have two parts: the inner product $a \cdot b$ is the symmetric part, while the wedge product $a \wedge b$ is the antisymmetric part.

$$
a b=a \cdot b+a \wedge b .
$$

The dimension of $G_{n=p, q, r}$ is $2^{n}$, and $G_{n}$ is constructed by the application of the geometric product over the vector basis $e_{i}$. This results in a basis for $G_{n}$ containing elements of different grade called blades (e.g. scalars, vectors, bivectors, trivectors, etc): $1, e_{1} \ldots e_{12} \ldots e_{123} \ldots I$, which is called basis blade; where the element of maximum grade is the pseudoscalar $I=e_{1} \wedge e_{2} \ldots \wedge e_{n}$. Given a multivector $M$ 
(linear combination of blades), if we are interested in extracting only the blades of a given grade, we write $\langle M\rangle_{r}$ where $r$ is the grade of the blades we want to extract (obtaining an homogeneous multivector $M^{\prime}$ or a $r$-vector).

\subsection{Conformal Geometric Algebra}

To work in Conformal Geometric Algebra (CGA) $G_{4,1,0}$ means to embed the Euclidean space in a higher dimensional space with two extra basis vectors which have particular meaning; in this way, we represent particular objects of the Euclidean space with subspaces of the conformal space. The vectors we add are $e_{+}$and $e_{-}$, which square to $1,-1$, respectively. With these two vectors, we define the null vectors

$$
e_{0}=\frac{1}{2}\left(e_{-}-e_{+}\right) ; \quad e_{\infty}=\left(e_{-}+e_{+}\right),
$$

interpreted as the origin and the point at infinity, respectively. From now and in the rest of the paper, points in the 3D-Euclidean space are represented in lowercase letters, while conformal points in uppercase letters. To map a point $x \in \mathcal{V}^{3}$ to the conformal space in $G_{4,1}$, we use

$$
X=x+\frac{1}{2} x^{2} e_{\infty}+e_{0}
$$

The table 1 shows the representation some the entities in CGA. All these entities and their transformations can be managed easily using the rigid motion operators described later.

Table 1. Standard representation of different entities in conformal geometric algebra

\begin{tabular}{|c|c||c|c|}
\hline Entity & Standard representation & Entity & Standard representation \\
\hline Sphere & $S=c+\frac{1}{2}\left(c^{2}-\rho^{2}\right) e_{\infty}+e_{0}$ & Point pair & $P P=S_{1} \wedge S_{2} \wedge S_{3}$ \\
\hline Point & $X=x+\frac{1}{2} x^{2} e_{\infty}+e_{0}$ & Circle & $Z=S_{1} \wedge S_{2}$ \\
\hline Line & $L=r I_{E}+e_{\infty} m I_{E}$ & Plane & $P=n I_{E}-d e_{\infty}$ \\
& $r=a-b$ & & $n=(a-b) \wedge(a-c)$ \\
& $m=a \wedge b$ & & $d=(a \wedge b \wedge c) I_{E}$ \\
\hline
\end{tabular}

In CGA, rotations are represented by the rotors, which are defined as

$$
R=e^{\frac{1}{2} \mathbf{b} \theta}=\cos \frac{\theta}{2}+\mathbf{b} \sin \frac{\theta}{2}
$$

where $\mathbf{b}$ is the bivector dual to the rotation axis, and $\theta$ is the rotation angle. Rotation of an entity is carried out by multiplying it by the left with the rotor $R$, and by the right for the reversion of the rotor $\tilde{R}: X^{\prime}=R X \tilde{R}$. Translation is carried out by the so called translator

$$
T=e^{\frac{e_{\infty} t}{2}}=1+\frac{e_{\infty} t}{2}
$$


where $t \in\left\langle G_{3}\right\rangle_{1}$ is the translation vector. Note that this operator can be interpreted as a special rotor, expressed in a null space because $e_{\text {infty }}^{2}=0$. Translations are applied in a similar way to rotations: $X^{\prime}=T X \tilde{T}$.

To express rigid body transformations, rotors and translators are applied consecutively. The result is called motor:

$$
M=T R
$$

Such operator is applied to any entity of any dimension by multiplying the entity by the operator from the left, and by the reverse of the operator from the right: $X^{\prime}=M X \tilde{M}$. The motor $M$ is a special multivector of even grade. To see its components, let us carry out the multiplication of $\mathrm{R}$ and $\mathrm{T}$

$$
\begin{aligned}
M & =T R \\
& =\left(1+\frac{1}{2} e_{\infty} t\right)\left(\cos \left(\frac{\theta}{2}\right)+\mathbf{b} \sin \left(\frac{\theta}{2}\right)\right) \\
& =\cos \left(\frac{\theta}{2}\right)+\mathbf{b} \sin \left(\frac{\theta}{2}\right)+\frac{1}{2} e_{\infty}\left(t\left(\cos \left(\frac{\theta}{2}\right)+\mathbf{b} \sin \left(\frac{\theta}{2}\right)\right)\right) \\
& =R+R^{\prime}
\end{aligned}
$$

Since the multiplication of a vector $t \in\left\langle G_{3}\right\rangle_{1}$ by a bivector $\mathbf{b} \in\left\langle G_{3}\right\rangle_{2}$ results in a multivector of the form $\lambda_{1} e_{1}+\lambda_{2} e_{2}+\lambda_{3} e_{3}+\lambda_{4} e_{123}$, and since $t \cos \left(\frac{\theta}{2}\right) \in\left\langle G_{3}\right\rangle_{1}$, we can rewrite (17) as

$$
\begin{aligned}
M & =\cos \left(\frac{\theta}{2}\right)+\mathbf{b} \sin \left(\frac{\theta}{2}\right)+\frac{1}{2} e_{\infty}\left(t \cos \left(\frac{\theta}{2}\right)+t \mathbf{b} \sin \left(\frac{\theta}{2}\right)\right) \\
& =\cos \left(\frac{\theta}{2}\right)+\mathbf{b} \sin \left(\frac{\theta}{2}\right)+\frac{1}{2} e_{\infty}\left(t \cos \left(\frac{\theta}{2}\right)+\lambda_{1} e_{1}+\lambda_{2} e_{2}+\lambda_{3} e_{3}+\lambda_{4} e_{123}\right. \\
& =\cos \left(\frac{\theta}{2}\right)+\mathbf{b} \sin \left(\frac{\theta}{2}\right)+e_{\infty}\left(t^{\prime}+\lambda e_{123}\right) \\
& \left.=\cos \left(\frac{\theta}{2}\right)+\mathbf{b} \sin \left(\frac{\theta}{2}\right)+e_{\infty} t^{\prime}+\lambda e_{\infty 123}\right)
\end{aligned}
$$

where $t^{\prime} \in\left\langle G_{3}\right\rangle_{1}$ and $\lambda=\frac{1}{2} \lambda_{4}$. Note that $e_{\infty} t^{\prime}$ is a bivector with components $e_{\infty 1}, e_{\infty 2}, e_{\infty 3}$. If we take only the bivectorial parts of the motor M, we obtain

$$
\begin{aligned}
\langle M\rangle_{2} & =\langle R\rangle_{2}+\left\langle R^{\prime}\right\rangle_{2} \\
& =\mathbf{m}+\mathbf{m}^{\prime} \\
& =\sin \left(\frac{\theta}{2}\right) \mathbf{b}+e_{\infty} t^{\prime}
\end{aligned}
$$

Therefore, if we express the vector $t^{\prime}$ in terms of their dual bivector $t^{\prime}=t^{\prime \prime} I_{E}$, we can rewrite (9) as

$$
\langle M\rangle_{2}=b^{\prime} I_{E}+e_{\infty} t^{\prime \prime} I_{E}
$$

If we see the representation of the lines in the table 1, we observe that the bivectorial part of the motor $\mathrm{M}$ is in fact a line and it corresponds to the screw axis in which is carried out the rotation and translation of the object. 


\section{The Endoscope-Polaris Calibration in CGA}

The hand-eye calibration is the calculation of the relative pose (position and orientation) between a robotic hand (arm) and a rigid camera mounted on it. Using this camera, we can determine the position in its coordinate system of an objective to catch or to reach; however, the commands of movements are in the coordinate system of the robotic hand (arm); therefore, to know the hand-eye transformation can be of great utility in this kind of tasks.

The usual way to describe the hand-eye calibration is by means of homogeneous transformation matrices [3] . In order to solve this problem, at least two movements are required with non parallel rotation axes, and several methods have been proposed to find the solution: some people estimate the rotation at first and later the translation [3, while others make it simultaneously [4. Daniilidis [5] presents a solution based on dual quaternions, while [6] proposes the use of the motor algebra $G_{3,0,1}$. This work formulates the problem in terms of motors of the conformal geometric algebra framework. Since motor algebra is a subalgebra of conformal algebra, we can also formulate in conformal geometric algebra the hand-eye problem making use of the motors in CGA. Following the formulation of [6] for the hand-eye calibration problem, it will be expressed as

$$
M_{A} M_{X}=M_{X} M_{B}
$$

where $M_{A}=A+A^{\prime}, M_{B}=B+B^{\prime}$ and $M_{X}=R+R^{\prime}$ (Sect. 2.1). In [6], it is shown that the problem is solved using the lines defined by the motors

$$
\begin{aligned}
L_{A} & =\mathbf{a}+\mathbf{a}^{\prime} \\
& =M_{X} L_{B} \tilde{M}_{X} \\
& =\left(R+R^{\prime}\right)\left(\mathbf{b}+\mathbf{b}^{\prime}\right)\left(\widetilde{R+R^{\prime}}\right) \\
& =R \mathbf{b} \tilde{R}+e_{\infty}\left(R \mathbf{b} \tilde{R}^{\prime}+R \mathbf{b}^{\prime} \tilde{R}+R^{\prime} \mathbf{b} \tilde{R}\right)
\end{aligned}
$$

where $\mathbf{a}, \mathbf{a}^{\prime}, \mathbf{b}, \mathbf{b}^{\prime}$ are bivectors (like in (9)). By separating the real part and the part multiplied by $e_{\infty}$, we have

$$
\begin{aligned}
\mathbf{a} & =R \mathbf{b} \tilde{R} \\
\mathbf{a}^{\prime} & =R \mathbf{b} \tilde{R}^{\prime}+R \mathbf{b}^{\prime} \tilde{R}+R^{\prime} \mathbf{b} \tilde{R}
\end{aligned}
$$

Multiplying from the right by $R$ and using the relationship $\tilde{R} R^{\prime}+\tilde{R}^{\prime} R=0$, the following relationships are obtained

$$
\begin{aligned}
\mathbf{a} R-R \mathbf{b} & =0 \\
\left(\mathbf{a}^{\prime} R-R \mathbf{b}^{\prime}\right)+\left(\mathbf{a} R^{\prime}-R^{\prime} \mathbf{b}\right) & =0
\end{aligned}
$$

which can be expressed in matrix form as

$$
\left[\begin{array}{ccc}
\mathbf{a}-\mathbf{b}[\mathbf{a}+\mathbf{b}]_{\times} & \mathbf{0}_{\mathbf{3} \times \mathbf{1}} & \mathbf{0}_{\mathbf{3} \times \mathbf{3}} \\
\mathbf{a}^{\prime}-\mathbf{b}^{\prime}\left[\mathbf{a}^{\prime}+\mathbf{b}^{\prime}\right]_{\times} \mathbf{a}-\mathbf{b}[\mathbf{a}+\mathbf{b}]_{\times}
\end{array}\right]\left[\begin{array}{c}
R \\
R^{\prime}
\end{array}\right]=0
$$


We call $\mathrm{D}$ to this $6 \times 8$ matrix; the unknown vector $\left[R, R^{\prime}\right]^{T}$ is 8 -dimensional. The notation $[u]_{\times}$represents the skew-symmetric matrix formed with the vector $u$. The matrix $D$ is composed only by bivectors (blades of any other grade are not included), therefore we can use the SVD method to find $\left[\begin{array}{ll}R & R^{\prime}\end{array}\right]^{T}$ as the kernel of $D$.

Considering that we have $n \geq 2$ movements, the following matrix is built

$$
C=\left[\begin{array}{lllll}
D_{1}^{T} & D_{2}^{T} & D_{3}^{T} & D_{4}^{T} & \cdots
\end{array}\right]^{T}
$$

in order to apply the SVD method and to find the solution for $\left[R, R^{\prime}\right]^{T}$. Since the range of the matrix $C$ is at most 6 , the last right two singular vectors, $v_{7}$ and $v_{8}$ correspond to the two singular values whose value is zero or near to zero, and such vectors expand the null space of $C$. Therefore, as $\left[R, R^{\prime}\right]^{T}$ is a null vector of $C$, we can express it as a linear combination of $v_{7}$ and $v_{8}$. If we express these vectors in terms of two vectors of $4 \mathrm{D} v_{7}=\left(u_{1}, v_{1}\right)^{T}$ and $v_{8}=\left(u_{2}, v_{2}\right)^{T}$, this linear combination can be expressed as

$$
\left[\begin{array}{l}
R \\
R^{\prime}
\end{array}\right]=\alpha\left[\begin{array}{l}
u_{1} \\
v_{1}^{\prime}
\end{array}\right]+\beta\left[\begin{array}{l}
u_{2} \\
v_{2}^{\prime}
\end{array}\right]
$$

Taking into account the geometric constraints

$$
R \tilde{R}=1 \quad \text { y } \quad \tilde{R} R^{\prime}+\tilde{R}^{\prime} R=0
$$

we obtain the following quadratic equations in terms of $\alpha$ and $\beta$

$$
\begin{aligned}
\alpha^{2} u_{1}^{T} u_{1}+2 \alpha \beta u_{1}^{T} u_{2}+\beta^{2} u_{2}^{T} u_{2} & =1 \\
\alpha^{2} u_{1}^{T} v_{1}+\alpha \beta\left(u_{1}^{T} v_{2}+u_{2}^{T} v_{1}\right)+\beta^{2} u_{2}^{T} v_{2} & =0
\end{aligned}
$$

In order to solve these equations, we make a change of variable, substituting in (22) $\mu=\alpha / \beta$ and we obtain two solutions for $\mu$. Going back to (21) and replacing the relationship $\alpha=\mu \beta$, we obtain

$$
\beta^{2}\left(\mu^{2} u_{1}^{T} u_{1}+\mu\left(2 u_{1}^{T} u_{2}\right)+u_{2}^{T} u_{2}=1\right.
$$

which takes two solutions for $\beta$.

The optical tracking system used is the Enhanced Hybrid Polaris System, which is labeled as (3) in the scenario shown in figure 1 This system emits infrared light that is reflected by markers placed to the object we are interested to track; the reflected light is detected by the sensors of the Polaris system, and then it estimates the 3D position of the different markers. We attach the local coordinate system of the markers to one of them. By this way, we know the transformation between the markers reference frame and the Polaris reference frame.

When using information obtained from endoscopy or neuro-sonography, we must relate what is been observed by the endoscopic camera (or ultrasound system), with the virtual model. To solve this problem, we propose the formulation 
of the hand-eye calibration problem in terms of the Conformal Geometric Algebra, to calculate the transformation between the coordinate system of the endoscopic camera, and the coordinate system of the spherical markers placed to the endoscope. We call this task the endoscope-Polaris calibration. The scenario is shown in figure 2, where the reader can see that there is a (rigid) transformation between the calibration grid, and the Polaris System, $\mathbf{M}_{\mathbf{B}_{\mathbf{g}}}$. Such transformation will be used to validate the results of the endoscope-Polaris calibration method. The transformations involved in the problem are expressed as motors of the

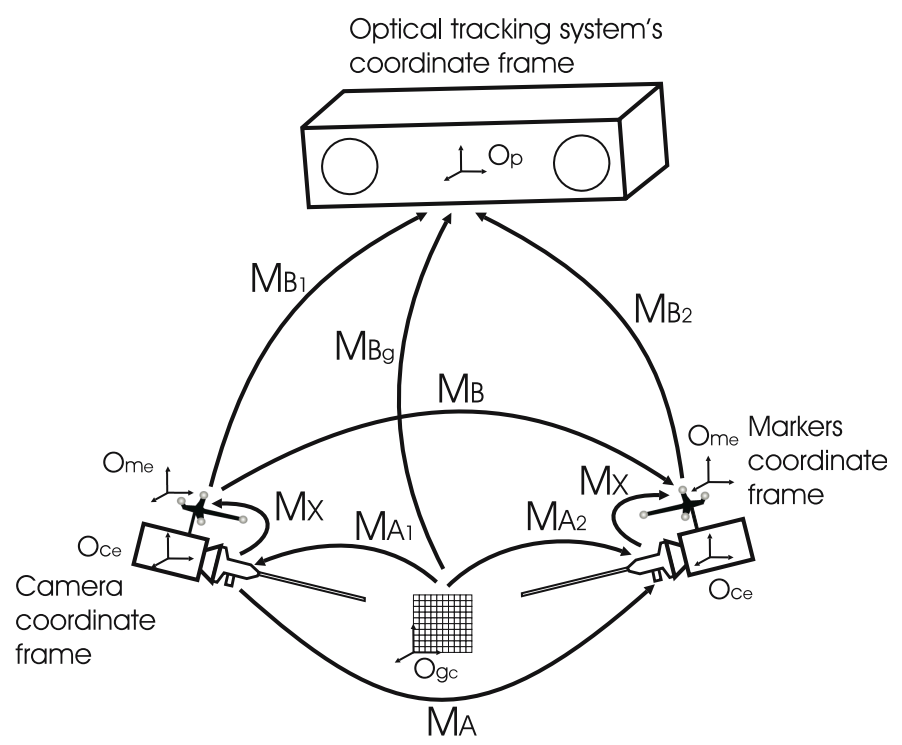

Fig. 2. The problem of the calibration between the endoscopic camera and the optical tracking system (Polaris)

CGA: $M=T R$.

The procedure is summarized as follows

1. Given $n$ movements of the endoscopic camera (we move it freely by hand to arbitrary positions), $M_{B_{i}}$, and their corresponding movements $M_{A_{i}}$, verify if their scalar parts are equal.

2. For the movements that fulfill the previous requirement, extract the directions and moments of the lines $L_{A_{i}}$ and $L_{B_{i}}$ defined by the motors. Build the matrix $C$ as in (18).

3. Apply SVD to matrix $C$. Take the right singular vectors $v_{7}$ and $v_{8}$ corresponding to the two singular values nearest to zero (a threshold is applied by the noise).

4. Compute the coefficients for (22) and find the two solutions of $\mu$. 
5. For both values of $\mu$, compute the value of $\mu^{2} u_{1}^{T} u_{1}+2 \mu u_{1}^{T} u_{2}+u_{2}^{T} u_{2}$ and choose the one that gives the biggest value. Then, compute $\alpha$ and $\beta$.

6. The final solution is $\alpha v_{7}+\beta v_{8}$

\section{Experimental Results}

In order to validate the accuracy of the estimated transformation $\mathbf{M}_{\mathbf{X}}$, we use the calibration grid used to calibrate the endoscopic camera by Zhang's method 7], and shown in Fig. 3, a. Let be $X_{g}$ the set of points corresponding to the corners of the calibration grid, referred to $\mathbf{O}_{\mathbf{g}_{\mathbf{c}}}$. These coordinates are expressed in millimeters, according to the size of each square in the calibration grid, which in our case has $1.25 \mathrm{~mm}$ by side.

1. Taking the points $X_{g}$ in the grid reference frame, apply the transformation $\mathbf{M}_{\mathbf{A}_{\mathbf{i}}}$ to express them in the camera's reference frame. Let be $X_{A_{i}}$ the resulting points.

2. Project the points $X_{A_{i}}$ to the image plane using

$$
x_{A_{i}}=K \quad\left[\begin{array}{ll}
R_{M_{A}} & t_{M_{A}}
\end{array}\right] X_{A_{i}}
$$

These points should be projected on the corners of the squares in the calibration grid on the image (Fig. 3).

3. Taking the points $X_{g}$, apply the transformations $\mathbf{M}_{\mathbf{B}_{\mathbf{g}}}, \mathbf{M}_{\mathbf{B}_{\mathbf{i}}}$ and $\mathbf{M}_{\mathbf{X}}$. Let be $X_{M_{B_{i} X}}$ the resulting points.

4. Project the points $X_{M_{B_{i} X}}$ onto the image plane using

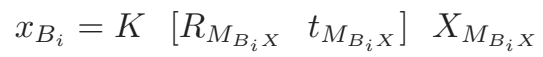

In the ideal case (without noise), the projected points $x_{A_{i}}$ should match with the projected points $x_{B_{i}}$. However, as a result of noise in the Polaris readings or noise in the estimation of transformations, takes place a small linear displacement between $x_{A_{i}}$ and $x_{B_{i}}$ (see Fig. 4.a). We can measure the error $\epsilon$ between the two projections as

$$
\epsilon=\frac{\sum_{i=1}^{n}\left(x_{A_{i}}-x_{B_{i}}\right)}{n}
$$
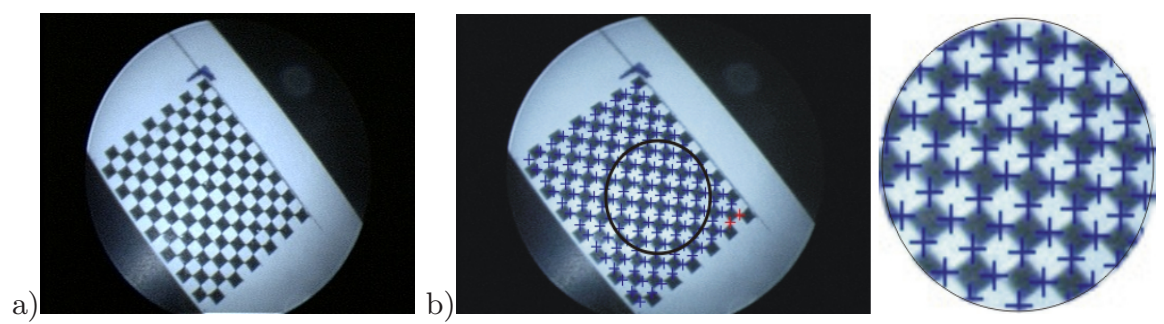

Fig. 3. a) Original image; b) Result of the projection using (24); it is included a zoom of the marked region for better visualization 

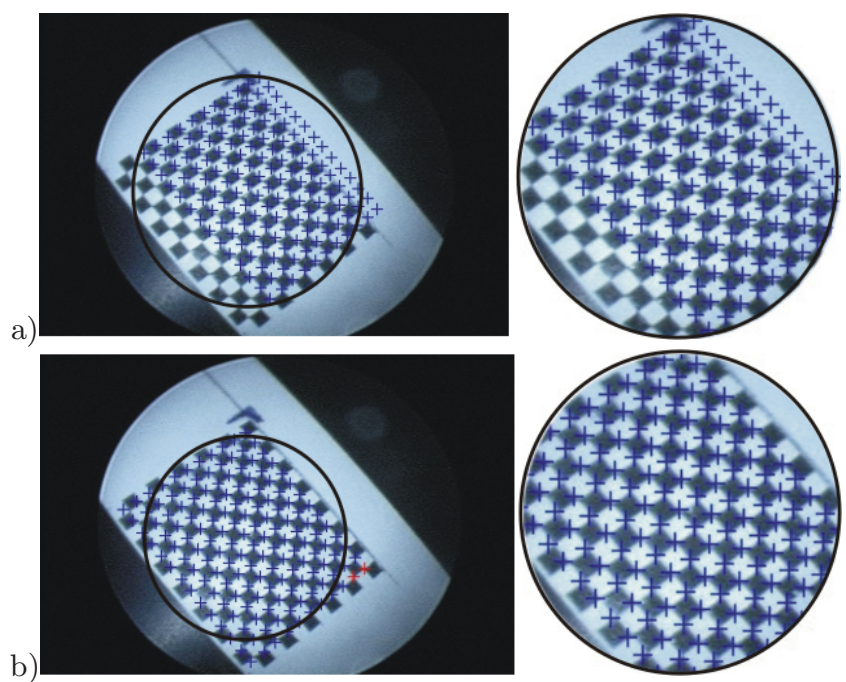

Fig. 4. a) Result of the projection using (25); b) Result applying (27)

5. In order to correct the displacement, the centroid of each point set is calculated: $c_{A_{i}}$ and $c_{B_{i}}$. Then, the points $x_{B_{i}}$ are displaced in such a way that the centroids match.

$$
x_{B_{i}}^{\prime}=x_{B_{i}}+\left(c_{A_{i}}-c_{B_{i}}\right)
$$

After the displacement, the average error is calculated as

$$
\epsilon^{\prime}=\frac{\sum_{i=1}^{n}\left(x_{A_{i}}-x_{B_{i}}^{\prime}\right)}{n}
$$

The figure 4, b shows the result after doing such correction to the points showed in figure 4 a.

The table 2 shows the errors measured for 9 different images without the telescope, while the table 3 shows the measured errors with 16 images with the telescope attached to the camera. Remember that the telescope is attached to the camera, then it is introduced to the patient's head in order to see and navigate inside the head.

Table 2. Average errors according to (27) and (28), measured in millimeters when projecting to the image plane the points $X_{g}$, according to (24) and (25) without the telescope attached to the camera

\begin{tabular}{|c||c|c||c||c|c||c||c|c|}
\hline Image & $\epsilon(\underline{26})$ & $\epsilon^{\prime}(\underline{28})$ & Image & $\epsilon(\underline{26})$ & $\epsilon^{\prime}(\underline{28})$ & Image & $\epsilon(\underline{26})$ & $\epsilon^{\prime}(\underline{28})$ \\
\hline 1 & 0.029 & 0.246 & 4 & 0.038 & 0.254 & 7 & 0.036 & 0.242 \\
\hline 2 & 0.029 & 0.291 & 5 & 0.054 & 0.273854 & 8 & 0.030 & 0.290 \\
\hline 3 & 0.029 & 0.327 & 6 & 0.026 & 0.285 & 9 & 0.030 & 0.263 \\
\hline
\end{tabular}


Table 3. Average errors according to (27) and (28), measured in millimeters when projecting to the image plane the points $X_{g}$ according to (24) and (25) attaching the telescope to the camera

\begin{tabular}{|c||c|c||c||c|c||c||c|c||c||c|c|}
\hline Image & $\epsilon(26)$ & $\epsilon^{\prime}(28)$ & Image & $\epsilon(26)$ & $\epsilon^{\prime}(28)$ & Image & $\epsilon(26)$ & $\epsilon^{\prime}(28)$ & Image & $\epsilon(26)$ & $\epsilon^{\prime}(28)$ \\
\hline 1 & 0.068 & 0.111 & 5 & 0.125 & 0.149 & 9 & 0.039 & 0.041 & 13 & 0.048 & 0.051 \\
\hline 2 & 0.067 & 0.222 & 6 & 0.057 & 0.146 & 10 & 0.244 & 0.258 & 14 & 0.031 & 0.103 \\
\hline 3 & 0.048 & 0.150 & 7 & 0.163 & 0.204 & 11 & 0.063 & 0.462 & 15 & 0.057 & 0.082 \\
\hline 4 & 0.076 & 0.162 & 8 & 0.075 & 0.090 & 12 & 0.048 & 0.142 & 16 & 0.033 & 0.1022 \\
\hline
\end{tabular}

\section{Conclusions}

In this work we have presented a method to estimate the transformation relating an endoscopic camera used in surgical procedures, with the set of spherical markers placed on it, which are tracked by an optical tracking system. By this way, we have all the transformations needed to appropriately compute a composed transformation relating the preoperative data (virtual model) with the intraoperative data obtained from the endoscopic camera.

The presented approach take advantage of the representation of rigid transformations (rotation and translation) in the conformal geometric algebra, which are expressed as versors called motors. The composition of such motors was analyzed, showing that they contain the line representing the screw axis in which is carried out the rotation and translation of the object. Then the transformation is estimated based on the lines defined by the motors in different movements of the endoscopic camera. We think that numerical results prove that the method is accurate enough and it is suitable to be used in real surgeries.

\section{References}

1. Bayro-Corrochano, E.: Robot perception and action using conformal geometry. In: Handbook of Geometric Computing. Applications in Pattern Recognition, Computer Vision, Neurocomputing and Robotics. ch. 13, Springer Verlag, Heidelberg (2005)

2. Rosenhahn, B., Sommer, G.: Pose Estimation in Conformal Geometric Algebra. Christian-Albrechts-University of Kiel, Technical Report No. 0206, pp. 13-36 (2002)

3. Tsai, R.Y., Lenz, R.K.: A New Technique for Fully Autonomous and Efficient 3D Robotics Hand/Eye Calibration. IEEE Transactions on Robotics and Automation 5(3), 345-358 (1989)

4. Horaud, R., Dornaika, F.: Hand-Eye Calibration. International Journal on Robotics Research 14(3), 195-210 (1995)

5. Daniilidis, K.: Hand-Eye Calibration Using Dual Quaternions. The International Journal of Robotics Research 18(3), 286-298 (1999)

6. Bayro-Corrochano, E., Sommer, G., Dannilidis, K.: Motor Algebra for 3D Kinematics: The Case of the Hand-Eye Calibration. Journal of Mathematical Imaging and Vision 13, 79-100 (2000)

7. Zhang, Z.: A Flexible New Technique for Camera Calibration. Microsoft Research $1-21(1999)$ 